OPEN ACCESS

Edited by:

John H. Gruzelier,

Goldsmiths, University of London, UK

Reviewed by:

Manousos A. Klados,

Max Planck Institute for Human Cognitive and Brain Sciences,

Germany

Human Friedrich Unterrainer,

University of Graz, Austria

${ }^{*}$ Correspondence:

Zhen Yuan

zhenyuan@umac.mo

Received: 08 January 2017 Accepted: 22 March 2017

Published: 06 April 2017

Citation:

leong HF and Yuan Z (2017)

Resting-State Neuroimaging and

Neuropsychological Findings in Opioid

Use Disorder during Abstinence: A

Review.

Front. Hum. Neurosci. 11:169. doi: 10.3389/fnhum.2017.00169

\section{Resting-State Neuroimaging and Neuropsychological Findings in Opioid Use Disorder during Abstinence: A Review}

\author{
Hada Fong-ha leong and Zhen Yuan* \\ Bioimaging Core, Faculty of Health Sciences, University of Macau, Taipa, Macau
}

Dependence to opiates, including illicit heroin and prescription pain killers, and treatment of the opioid use disorder (OUD) have been longstanding problems over the world. Despite intense efforts to scientific investigation and public health care, treatment outcomes have not significantly improved for the past 50 years. One reason behind the continuing use of heroin worldwide despite such efforts is its highly addictive nature. Brain imaging studies over the past two decades have made significant contribution to the understanding of the addictive properties as to be due in part to biological processes, specifically those in the brain structure and function. Moreover, traditional clinical neuropsychology studies also contribute to the account in part for the treatmentrefractory nature of the drug abuse. However, there is a gap between those studies, and the rates of relapse are still high. Thus, a multidisciplinary approach is needed to understand the fundamental neural mechanism of OUD. How does the brain of an OUD patient functionally and cognitively differ from others? This brief review is to compare and contrast the current literature on non-invasive resting state neuroimaging and clinical neuropsychological studies with the focus on the abstinence stage in OUD. The results show as follow:

1. Brain connectivity strength in the reward system, dysregulation of circuits associated with emotion and stress, enhanced beta and alpha power activity, and high impulsivity are induced by OUD.

2. Some recovery signs in cognition are demonstrated in OUD subjects after prolonged abstinence, but not in the subjects undergoing methadone treatment.

3. Normalization in the composition of brain oscillations especially in the temporal region is induced and restored by methadone treatment in roughly 6 months in mean duration for OUDs having a mean opioid-use history of 10 years.

We hope that the review provides valuable implications for clinical research and practice and paves a new insight into the future path to the identification of potential biomarkers and clinical outcome predictors in OUD in the domains of brain regions, functions, and behaviors.

Keywords: heroin, opioid, electroencephalography, functional magnetic resonance imaging, neuropsychological tests, resting-state functional connectivity, cognition, addiction 


\section{INTRODUCTION}

Among the abused substances, opiates including illicit heroin and prescription opiates are one of the most frequently abused and powerfully dependent drugs. Opioid use disorder (OUD) has been associated with severe and dire global and societal consequences. Overdose is a major cause of death (Levi et al., 2012), and the prevalence of blood borne viruses, such as HIV and hepatitis $\mathrm{C}$, are higher in illicit opiate users than the general population (Bart, 2012; United Nations Office on Drugs and Crime, 2014). The social burden due to OUD is tremendous but current pharmacological treatments of opioid-related conditions and psychological therapies are still insufficient to reduce the high relapse rate.

Substance use disorders (SUDs) are characterized as the compulsion of drug-seeking and drug-taking behaviors despite of harmful consequences, and it encircles a relapsing cycle of intoxication, binging, withdrawal and craving during abstinence (Goldstein and Volkow, 2011). It becomes the notion that the loss of control over drug consumption involves disruption of the reward mesocorticolimbic (MCL) circuits. However, how different substances of abuse manipulate particular brain regions within the circuits and to what extend the affected regions are related to certain cognition and behaviors need further elucidation.

Neuroimaging findings and clinical implications have provided convincing evidence that chronic opiate abuse affects the prefrontal cortex (PFC) (Parvaz et al., 2011), temporal insula and thalamus(Goldstein and Volkow, 2002), nucleus accumbens (Noel and Gratton, 1995), amygdala (Baxter et al., 2000), and sensorimotor cortices (Liu et al., 2009). However, the existing findings have not yet been sufficient to capture the integrity of the physiological events (i.e., brain regions) and psychological events (i.e., brain functions, and behaviors) in OUD. Examining how these two domains are entangled is essential to understand the fundamentals of neural mechanism aiming to improve clinical outcomes in the disorder.

Being able to remain abstinent is a positive sign of a clinical outcome (Reilly et al., 1995; Schuckit, 2016), especially for individuals with OUD of which the relapse rate has been the highest among SUDs (SAMHSA). The neural characteristics of OUD individuals regarding to the physiological and psychological events may vary in different stages of the relapsing cycle. Here we provide a review of the chronic effects of opiate focusing on OUD individuals who stayed abstinent for more than 2 weeks-when the acute withdrawal is subside. Particular emphasis is placed on the findings of two restingstate neuroimaging: (1) the high spatial resolution technique functional magnetic resonance imaging (fMRI), and (2) the high temporal resolution technique electroencephalography (EEG), as well as the evidences of neuropsychological performances.

The aim is to highlight the important aspects of integrating connectivity between brain regions, brain functions and behaviors in OUD. The joint approaches of resting-state neuroimaging and neuropsychology are suggested to increase the effectiveness of intervention during the abstinence stage for OUD long-term recovery. We hope the review make implication to the identification of potential biomarkers to prevent relapse.

\section{METHODOLOGICAL APPROACHES}

The available literature on OUD during abstinence was identified by means of regular searches in online electronic databases through the ISI WEB of Science. The scope of the current review was focused on human non-invasive fMRI and EEG resting-state neuroimaging and neuropsychological approaches to OUD studies during the abstinence period. The basic search terms included opiate, opioid, heroin, prescription opiates, morphine, dependence, addiction, abstinence. The selected neuroimaging studies used fMRI and EEG as an evaluation tool for assessing the brain activity differences among OUD subjects and control subjects. In addition to the basic search terms, the following search terms: resting state, functional connectivity, fMRI, EEG, were included. The fMRI studies related to structural connectivity were not considered in the review. The selected cognition and behavior studies on the other hand used neuropsychological assessments as an evaluation tool for assessing the performance differences between the study and control groups. In addition to the basic search terms, the following terms: cognition, task, neuropsychology, behavior, were searched. The articles from 1969 until November 2016 were included. Inclusion criteria for both neuroimaging and neuropsychological approaches for the study group were: (1) the OUD subjects meeting the standard (DSM IV axis 1, ICD-10) criteria, and (2) the subjects stayed abstinence for a minimum of 2 weeks. Because comorbidity, such as dependence to other substances, is not uncommon in OUD individuals, the concurrent medical patterns of the studies and treatment programs were documented in the review. However, studies of substances other than opiates as the primary drug of dependence were not considered in the review.

\section{NEUROIMAGING: RESTING-STATE FUNCTIONAL CONNECTIVITY (rsFC)}

Functional connectivity (FC) is a statistical dependence between the time series of measured neurophysiological signals (Gerstein and Perkel, 1969; Sporns et al., 2004). The basic concept is that two locations of a brain are considered to be functionally connected if they have coherent or synchronize dynamics. And the interpretation of different measures in the neurophysiological signals depends on the type of recordings being analyzed. Current advances in assessing brain network through restingstate functional connectivity (rsFC) using fMRI may allow for such system-level assessments and using EEG may allow for celllevel assessments. For example, a statistical dependence between neural time series in hemodynamic signals measured with fMRI could offer an interpretation into neuronal communication in the system between brain regions, whereas correlations in the spiking output of neurons measured with EEG could provide an interpretation as having functional interactions between neurons-embodied in the concept of a cell assembly, proposed 
by Hebb (2005). Emerging evidence supports the theory that circuits synchronized at rest are constrained to known, direct or polysynaptic anatomically interconnected regions (Damoiseaux and Greicius, 2009; Greicius et al., 2009; Honey et al., 2009), thus constituting plausible functional networks (Sutherland et al., 2012). More importantly, the strength of these resting-state networks could predict both behavioral accuracy and subsequent activation of the same brain areas when performing cognitive tasks (Sutherland et al., 2012). Therefore, alteration in rsFC can potentially be helpful to assess various neuropsychiatric trajectories in SUDs. The main goal of brain activity studies in SUDs is to assess the neurophysiological changes among the individuals dependent on the substances. Over the past several decades, non-invasive neuroimaging has contributed significant evidences into the neuroplastic adaptations that resulted from chronic drug intake. This review focuses on the studies of the rsFC generated by two powerful modalities of neurophysiological imaging: fMRI and EEG.

\section{RESTING-STATE FUNCTIONAL MAGNETIC RESONANCE IMAGING (fMRI)}

fMRI is a neuroimaging technique indirectly measuring brain activity by detecting neurophysiological changes in the cerebral blood flow and thus predicting neuronal activation in an area of the brain. Despite of the raising promise that rsFC may serve as a potential diagnostic tool for various mental disorders, rsFC has been applied in only a handful of opiate-related studies (Sutherland et al., 2012). Table 1 shows the summary of current findings of resting-state fMRI studies in the review.

First, alterations in rsFC strength between ventral striatum and various subcortical and cortical regions in the reward system have been observed. In the reward (MCL) circuits, the increased dopamine releases are thought to underlie the initial reinforcing influences of abused drugs (Nestler, 2005). Ma et al. (2010) tested the hypothesis whether or not the MCL circuits were altered in 12 abstinent heroin-using OUD subjects under methadone maintenance treatment (MMT) and 2 abstinent male heroinusing OUD subjects without any treatment and compared with 13 demographic-matched smokers. Results reported enhanced rsFC between the MCL regions and subcortical and cortical areas. In the MCL system, stronger rsFC between nucleus accumben and the ventral areas of medial (PFC), including rostral anterior cingulate cortex (CC) and medial orbitofrontal cortex (OFC) were observed, suggesting that connectivity within reward and motivation circuits may help the interpretation of altered incentive salience for drugs and drug-associated cue. Without considering the smoking habit, the finding of Zhai et al. (2015) was consistent with that of Ma et al. (2010), while Wang et al. (2016), on the other hand, showed reduction in rsFC between MCL regions and subcortical and cortical areas. It was noted that the reported abstinence duration of the OUD subjects underwent MMT (daily dose: $52.0 \pm 29.4 \mathrm{mg}$ ) in Wang (Wang et al., 2016) study was relatively longer than that of the OUD subjects in Ma (Ma et al., 2010), where the daily dose of MMT was not reported, and that of the OUD subjects in
Zhai (Zhai et al., 2015), where the treatment program was not reported in the study. It is not known if these factors (i.e., abstinence length, methadone maintenance length and dosage, and treatment program) played a role in the inconsistency of the rsFC alternation. Thus, more studies are needed to illustrate the hypothesis.

Second, altered connectivity between the circuits associated with emotion and stress regulations, including amygdala and insula, has been observed in OUD patients during multiple stages of dependency. Ma et al. $(2010,2011)$ reported increased rsFC between amygdala and lateral OFC. Similarly, Liu et al. (2009) reported increased rsFC strength in amygdala, insula, hippocampus, ventral striatum, supplementary motor area (SMA), anterior CC, and inferior frontal gyrus in the PFC in 12 abstinent male heroin-using OUD subjects compared with 12 age-matched controls. These regions were also identified as distinctive to access resting-state brain activities in heroin abstinent OUD subjects (Zhang et al., 2011), and the connectivity strengths in amygdala and insula were also observed in Xie et al. (2011). However, in a sample of chronic heroin-using OUD subjects $(n=30)$ underwent MMT, Wang et al. (2016) observed significant reduction in rsFC between insula and inferior OFC, amygdala, putamen, and caudate areas, where the reduced interhemispheric connectivity between these regions may predict a higher incidence of a failure of the integration process-a deviation from the normal neurodevelopmental trajectory (Fair et al., 2007, 2008). Taken together, further investigations in a larger sample $(n>30)$ are required to examine the alternation in the amygdala and insula networks in OUD when working under the assumptions that amygdala and its connected regions are critical neural substrates mediating continued drug intake, and that the neural interactions are essential to process processing emotional stimuli, generating affective states, and regulating emotion (Phillips et al., 2003; Pezawas et al., 2005; Stein et al., 2007).

Third, abnormal connectivity between the neural systems associated with cognitive control brain network, including the anterior CC, lateral PFC and parietal areas, has also been found in abstinent heroin-using OUD subjects. Yuan et al. (2010) found reduced $\mathrm{rsFC}$ strength between the left inferior parietal lobe and right dorsolateral PFC in 11 abstinent heroin-using OUD subjects underwent MMT comparing with 11 age-matched controls. Consistent to the hypothesis that rsFC is reduced between the anterior CC and dorsolateral PFC in abstinent heroin-using OUD subjects. Ma et al. (2011) also reported the reduction between the right dorso-anterior CC and left caudate.

\section{RESTING-STATE ELECTROENCEPHALOGRAPHY (EEG)}

The abovementioned cell assembly concept (Hebb, 2005) refers to a mechanism by which a coherent entity through the joint activity of distributed and interconnected neurons exhibits distinct features of a stimulus. In fact, neuronal inhibition plays an essential role in establishing these cell assembly, allowing the precise alignment of the spiking activity of neurons 


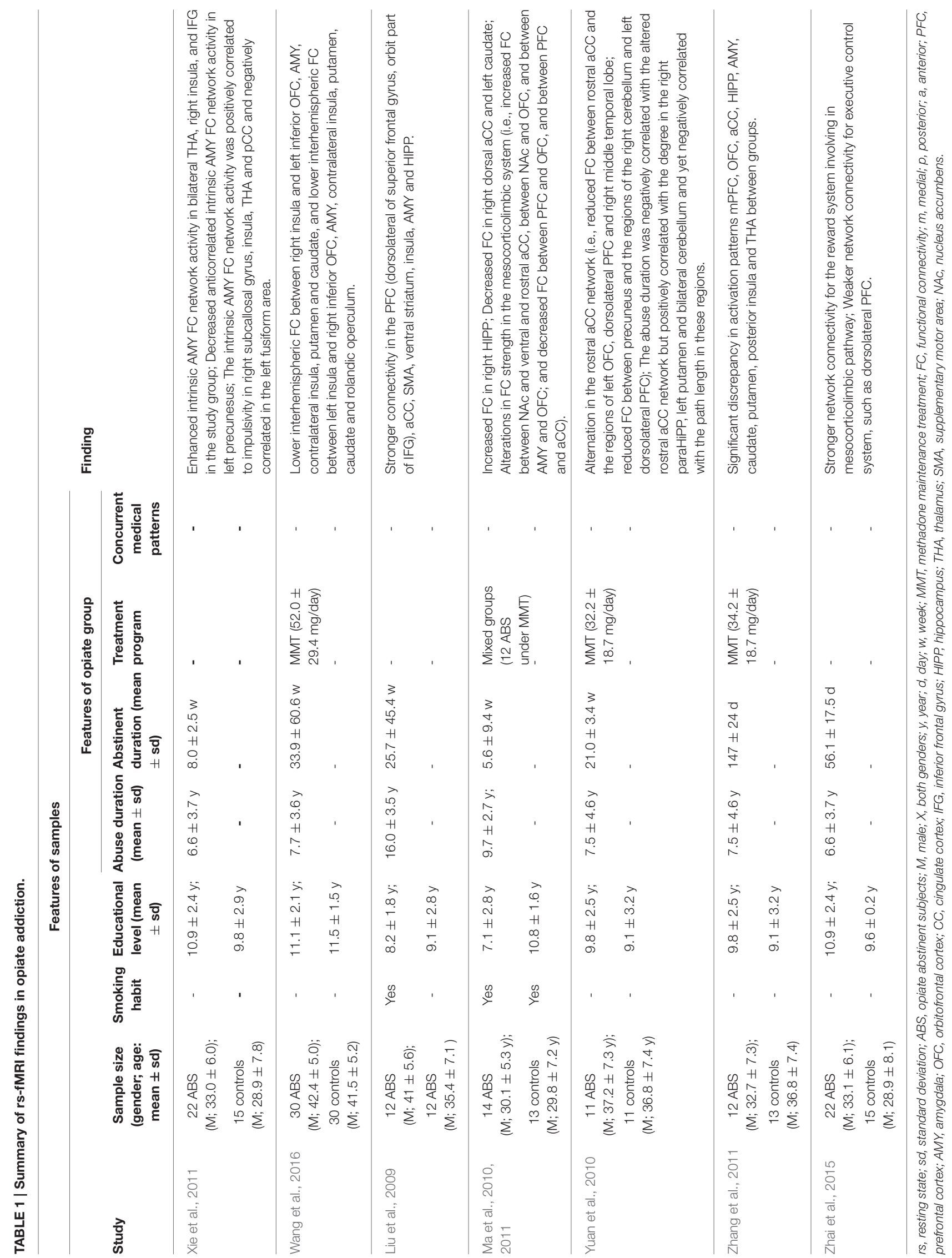


within specific time windows (Singer, 2013). In particular, oscillations between populations of neurons are synchronized by the precisely timed inhibitory signals, and thus fluctuations in neuronal excitability are coordinated (Singer, 1999; Fries et al., 2001; Womelsdorf et al., 2014). Human EEG is a neuroimaging technique that directly monitors and records the electrical activity (i.e., the coordinated oscillatory activity thought to mediate neuronal communication at cellular level) of the cerebral cortex. Scalp EEG activity demonstrates oscillations at different frequencies, categorized in frequency bands. Several of these oscillations have specific spatial distributions and are associated with different states of brain function, such as eye-closed resting state (Knyazeva and Innocenti, 2001). This neuroelectrophysiological approach has been used in restingstate heroin-using OUD studies. Table 2 demonstrates the summary of key findings of resting-state EEG studies in the review.

Fingelkurts et al. (2006a,b, 2007, 2009) examined the local and remote rsFC and the composition of brain oscillations in heroin abusers in longitudinal studies using 20 EEG channels. First, the research group (Fingelkurts et al., 2006a) studied 22 mixed-gender subjects with 4-26 years of heroin-use history who were at the stage of long-term abstinence and underwent MMT, as well as the OUD subjects who were at short-term withdrawal period and compared with 14 demographic-matched controls. Later they (Fingelkurts et al., 2007) focused on the 13 subjects out of the 22 heroin-using OUD subjects who were at the withdrawal period and compared with the 14 controls to examine the differences. It was found that both the long-term abstinent and short-term withdrawal OUD patients demonstrated an increase in local rsFC and temporal stabilization for alpha and beta bands across the cortex (Fingelkurts et al., 2006a), and only the short-term withdrawal OUD subjects displayed an increase in remote rsFC (Fingelkurts et al., 2007). Further, the research group (Fingelkurts et al., 2009) studied 6 abstinent subjects underwent MMT and compared with the same controls. It was discovered that methadone restored local and remote rsFCs in heroin-using OUD subjects. Moreover, the average amplitude of alpha and beta bands increased in the left frontal and right temporal cortices and decreased in the right parietal region. The average length of EEG segments was shorter in the right central for alpha and beta bands, right parietal for beta band, and midline occipital cortex for alpha band. Based on the rsEEG literature, these longitudinal observations concluded that 6-month MMT moderated distribution of rsFC of alpha and beta bands, normalized the composition of EEG activities, and restored the temporal structure of brain oscillations.

Gorricho and Usón (2008) evaluated the acute effect of methadone among 15 mix-gender subjects underwent chronic MMT, measuring the qEEG before and $3 \mathrm{~h}$ after the daily methadone treatment. Results showed that an increase in the slow-wave activity (delta and theta bands) and a decrease in the alpha activity of MMT subjects. Further, Franken (Franken et al., 2004) found that increased relative beta- 2 power and increased left intrahemispheric gamma coherence in the temporal and frontal regions in OUD when evaluating EEG power and coherence and brain functionality of 18 male heroin-using OUD subjects and compared with 12 healthy controls. The EEG coherence also showed correlations with clinical measures, such as chronic heroin craving, and having heroin-related thoughts, suggesting that the alterations were methadone-related.

\section{NEUROPSYCHOLOGY: COGNITIVE AND BEHAVIORAL MEASURES}

Neuropsychology is a discipline that seeks to understand the interrelationship of a human brain and mind in terms of behavioral science. Neuropsychological testing aims to assess the cognitive functioning of OUD subjects, as well as to determine the nature and extent of possible organic cerebral impairment which may be resulted from cumulative effects of chronic drug use on the central nervous system. By examining the performance patterns, which may characterize and distinguish certain SUD individuals, the neuropsychological assessment or task can serve as a tool to discern functional and clinical relationships between different psychological and cognitive processes, such as memory and reasoning (Miller, 1985). It is traditionally useful in contributing to therapeutic management, as well as in predicting treatment outcome. Table 3 summarizes the main findings of clinical neuropsychological research in OUD.

Opiate abstinent abusers have been compared to an extensive neuropsychological battery to normal controls (Zhao et al., 2009; Xie et al., 2011; Gerra et al., 2014; Morie et al., 2014; Yan et al., 2014; Zhai et al., 2015; Hou et al., 2016), to patients undergoing treatment program, such as MMT (Darke et al., 2000; Mintzer and Stitzer, 2002; Mintzer et al., 2005; Passetti et al., 2008), and subjects with known mental disorder, such as anxiety and depression (Zhou et al., 2012). These studies yielded some consistent results. First, OUD subjects showed significantly higher impulsivity in terms of attention/cognitive and nonplanning, even after opiate use ceases (Xie et al., 2011; Zhai et al., 2015; Hou et al., 2016). Interestingly, when the abstinent study group compared to the control group who were smokers, there was no significant difference in motor impulsivity, whereas there was significance when compared to healthy control.

Second, emotional processes, such as regulating stress and negative emotion, were interrupted after prolonged opiate intake. For example, Zhao et al. (2009) showed that acute stress at the time of recall enhanced retrieval of positively valenced words but no effect on negative and neutral word retrieval in abstinent subjects, suggesting the dysregulation of subjects' emotional memory processing under stress and thus providing insight into designing better treatment for patients with OUD. In addition, both Gerra et al. (2014) and Zhou et al. (2012) showed that abstinent OUD subjects presented highly sensitivity toward negative emotion. Gerra et al. (2014) further showed that the high basal anxiety level in OUD subjects and the severity of drug dependence were highly associated with their adverse childhood experiences.

Third, signs of recovery in cognitive performance were shown after sustained opiate abstinence. For example, when Darke et al. (2000) and Mintzer et al. (Mintzer and Stitzer, 2002; Mintzer et al., 2005) used a variety of neuropsychological tests 


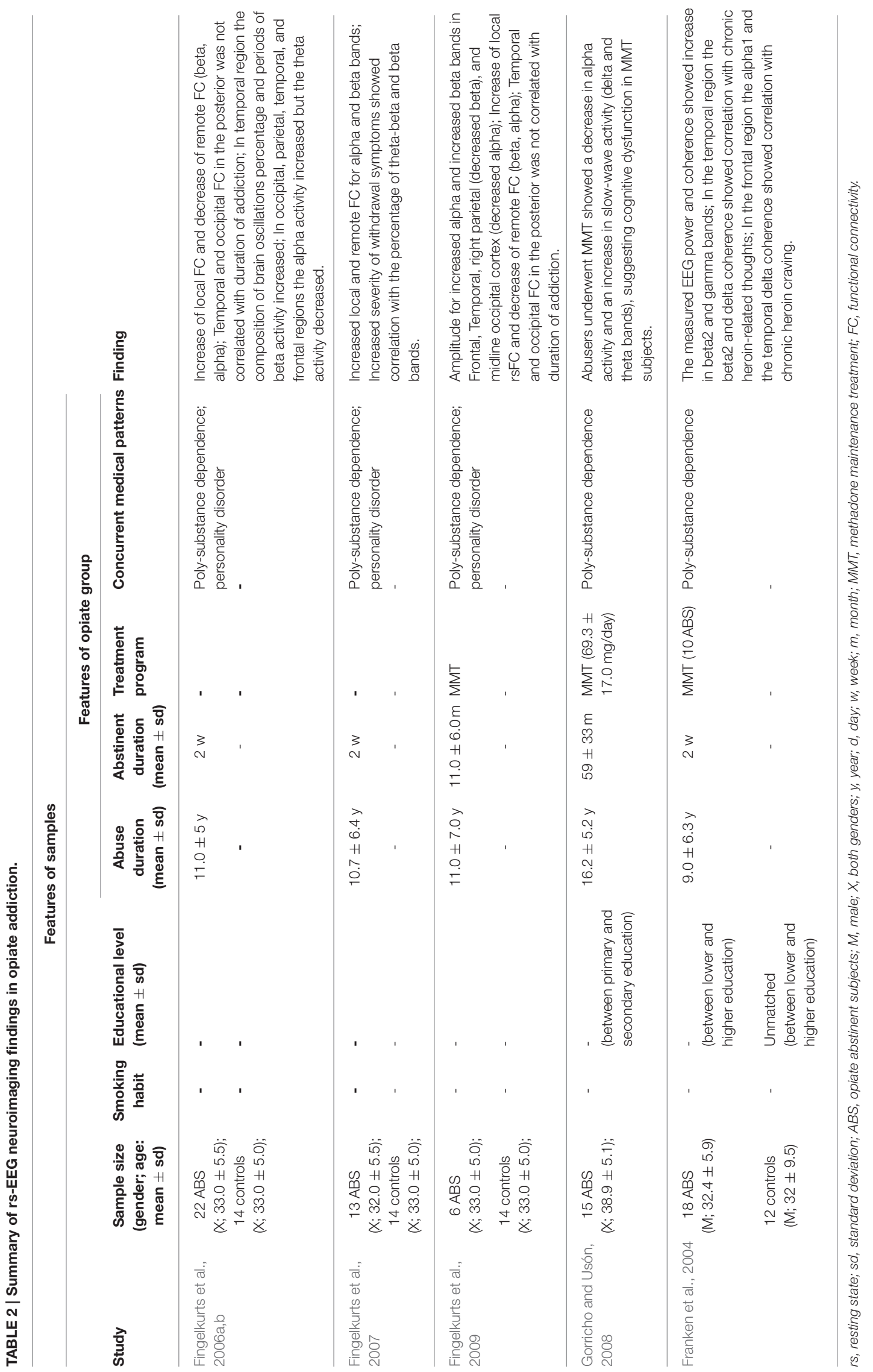




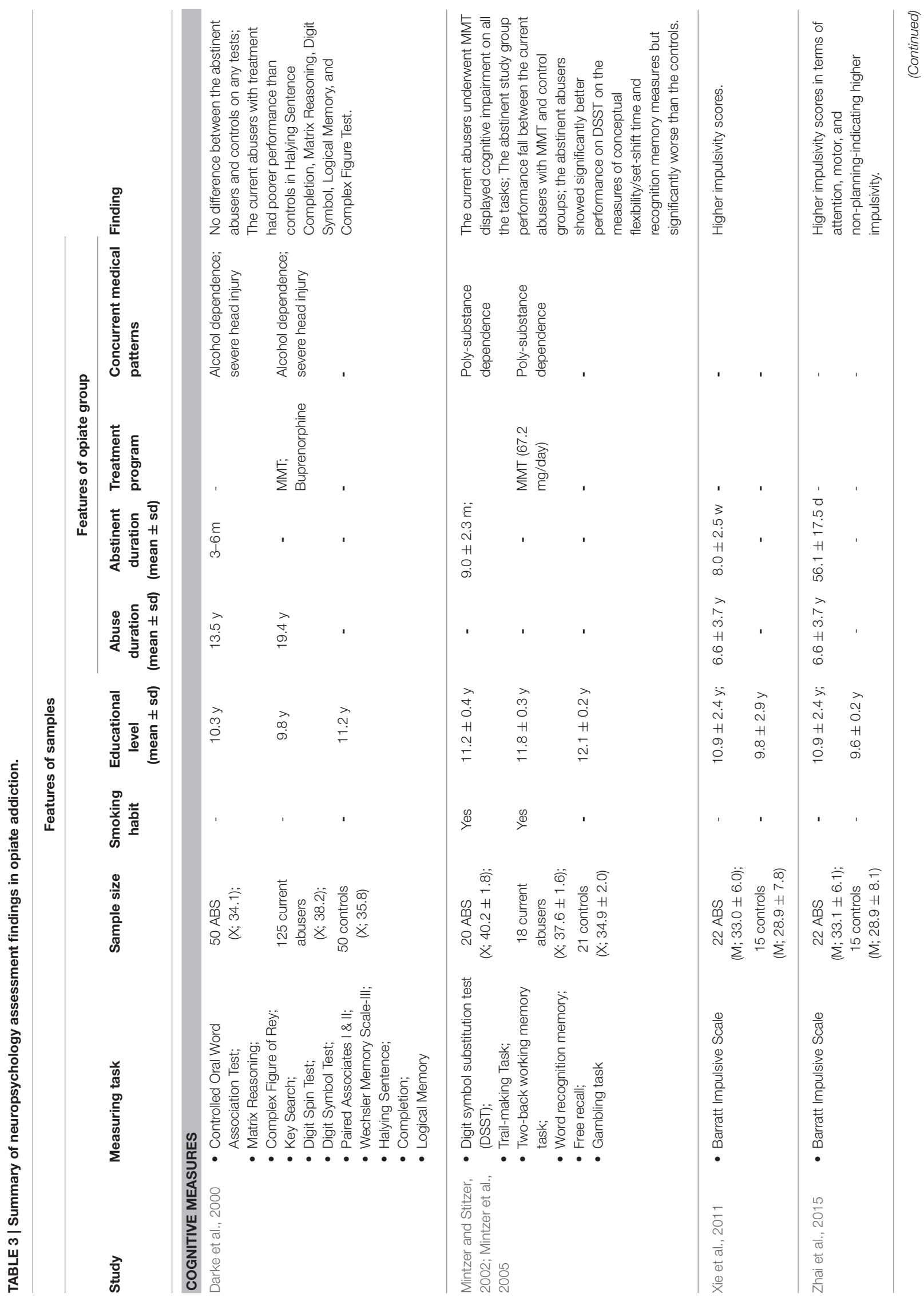




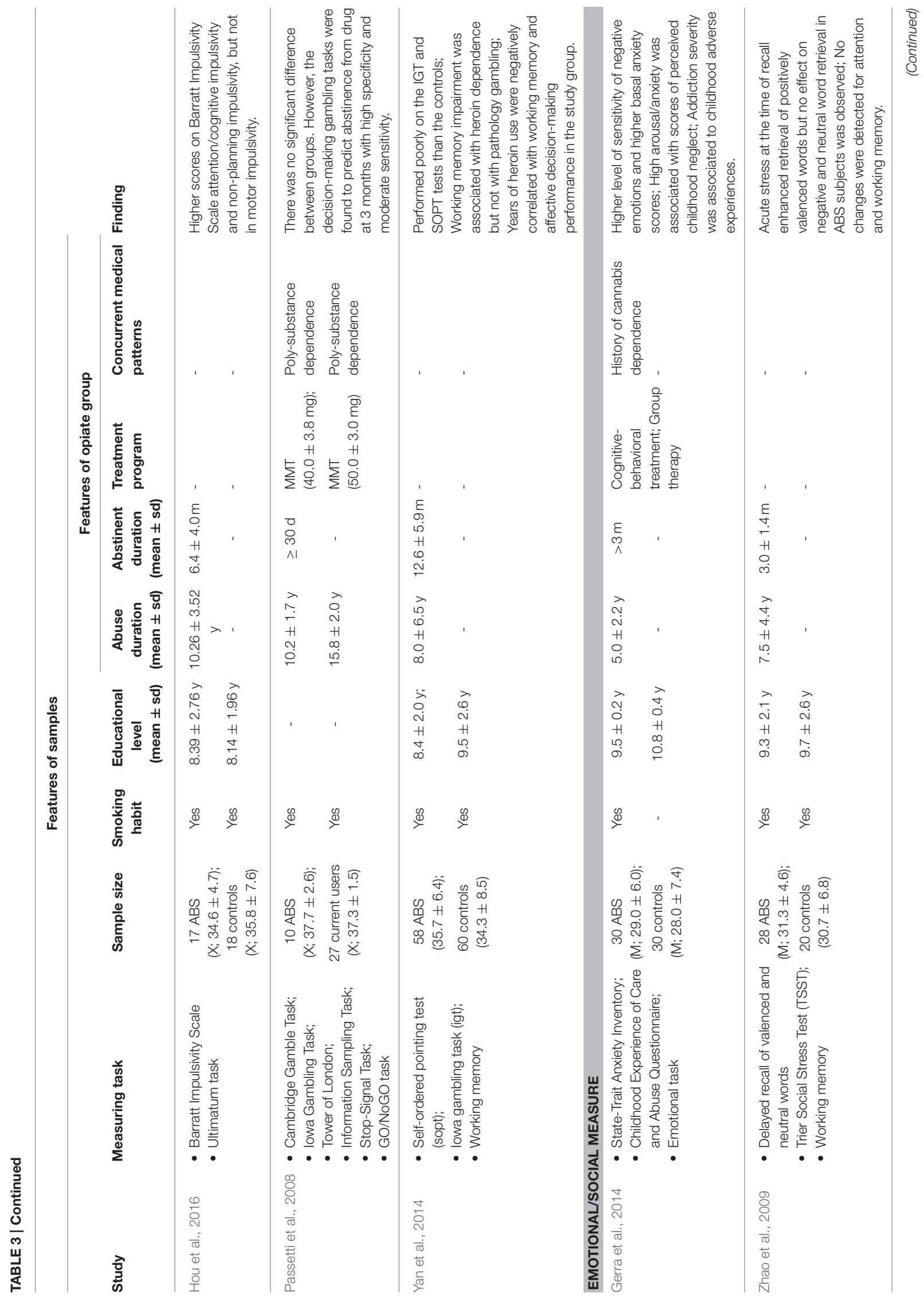




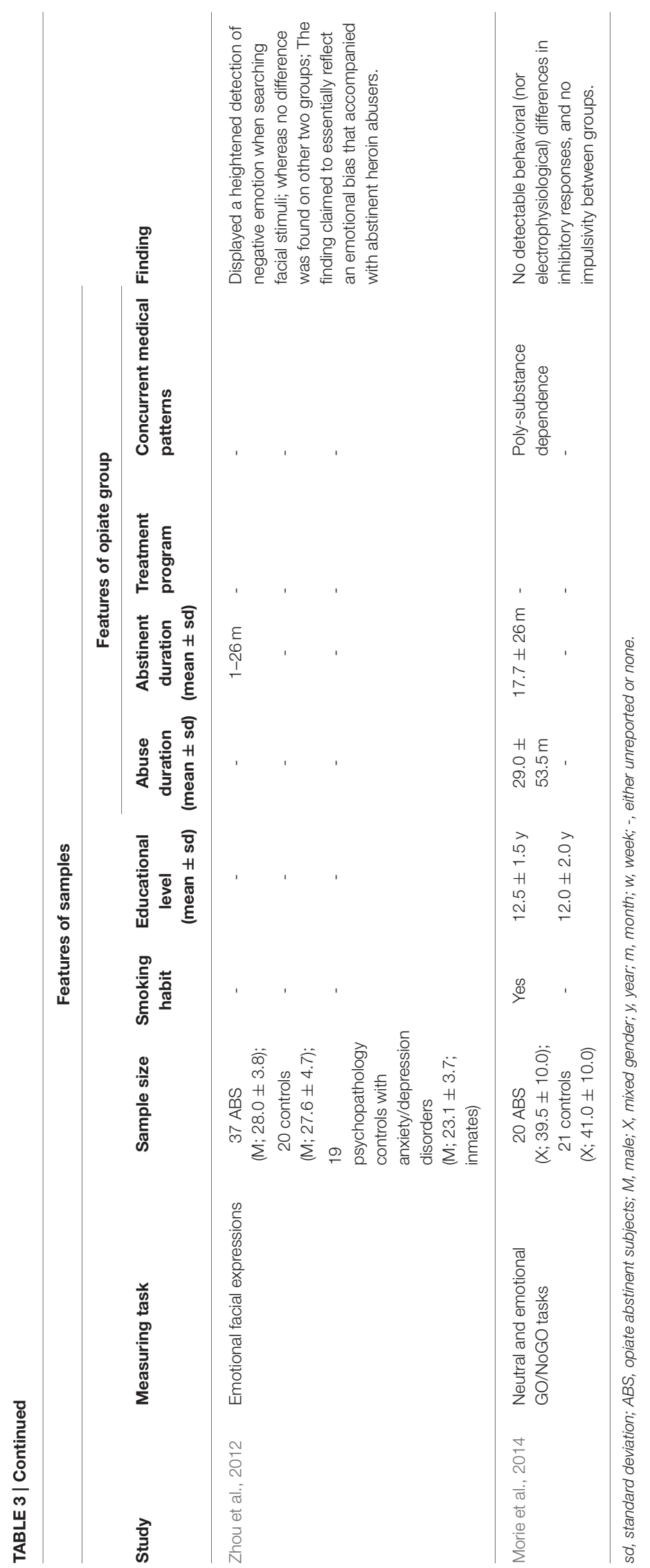


to assess the cognitive processes of abstinent OUD subjects and compare with both current opioid-using subjects and healthy controls, they found that there was no significant difference between abstinent OUD subjects and healthy controls, and yet the current opioid-using subjects underwent MMT demonstrated significantly poor performance, both studies suggesting that methadone and/or the acute effect of opiate might be associated with cognitive impairment, resulting in long-term opiate consumption. This assumption is also supported by the aforementioned neuroimaging findings (Gorricho and Usón, 2008; Fingelkurts et al., 2009; Ieong, 2013).

\section{LIMITATIONS AND FUTURE DIRECTION}

There are several important limitations in the review worth highlighting. First, the current review focuses on the functional connectivity during resting-state on the brain regions in OUD, but there are other forms of brain connectivity, including functional connectivity during task performance and structural connectivity. It remains unknown whether or not the functional connectivity is directly related to cognitive brain functions or the structural connectivity in OUD during abstinence. Future studies may be needed to further investigate and resolve these gaps. Indeed, the relationship between functional network and cognitive function is an essential question that has so far been relatively under-explored (Fornito et al., 2016). Initial work has shown that inter-individual variations in topological properties of functional networks correlated with variability in cognitive performance (Li et al., 2009; Bassett et al., 2011; Zalesky et al., 2011; Crossley et al., 2013, 2014). And thus, in addition to explore brain function network using neuroimaging techniques, exploring the cognitive relevance of the brain areas using neuropsychological testing and observing from clinical symptoms are essential to better understand OUD among SUDs and effectively identify and treat one OUD patient among other patients with SUDs.

Second, although we provide a comprehensive overview of resting-state neuroimaging and neuropsychological studies, the samples sizes are relative small, and the study groups are heterogeneous. It is noted that some of the study groups were with concurrent comorbidity or underwent different treatment programs, such as MMT, buprenorphine maintenance, and group therapy in the review. Although methadone, a full opioid agonist, and buprenorphine, a mixed opioid agonist antagonist, have been the mostly administrated drugs to ease OUD withdrawal symptoms (Schuckit, 2016), both of them bind to the opioid receptors in the brain to produce long-acting effects to relieve symptoms during abstinence when the short-acting opioids, such as heroin and prescription morphine, are discontinued. The current review has not fully addressed the treatment outcomes of these opioids, as well as the possible neural complications that may intervene the brain activities in terms of brain regions, functions and behaviors in OUD. In order to compensate the limitation of heterogeneity and facilitate future studies, the considerably important factors, such as the treatment programs, daily dose of MMT, smoking habit, education levels, and concurrent medical patterns of the subjects reported in the literature were detailed and tabled in the review. Large samples with matched controls should also be considered to confirm the findings and resolve the heterogeneity.

Third, we only briefly explored the social cognitive processes in OUD subjects because empirical studies are limited. Living in a complex social environment, one's decision-making often takes place in a social setting and is influenced by one's social cognitive abilities (Bargh, 1989) and personal experiences from childhood to adulthood (Chapman et al., 2004). To date, socio-cognitive process has not been fully investigated in an experimental research setting in OUD. We suggest the use of the powerful tools in neuroimaging and neuropsychology to further explore the chronic effect of opiate on cognitive and emotional processes in a social interactive environment or setting.

Further, in addition to the relatively high-cost fMRI to measure the hemodynamic responses, future studies can consider the alternative, non-invasive, rapid-growing and cost-effective functional near-infrared spectroscopy (fNIRS) as a neuroimaging tool to study functional connectivity in OUD (Ieong, 2015).

\section{CONCLUSION}

Recovery requires more than abstinence in OUD. Maintaining abstinence may be considered one step toward long-term recovery. The primary aim of the review was to present the current and comprehensive key findings using neuroimaging and clinical neuropsychological tools to explore the chronic influence of opiate during abstinence. We summarized that OUD subjects who stayed abstinence for 2 weeks or above demonstrated: (1) strengthened brain connectivity in the reward system and enhanced beta and alpha power activity, (2) recovery signs in several cognitive and behavioral performances but not in impulsivity, and (3) normalization of brain oscillations in the temporal area induced by methadone treatment in approximately 6 months and yet showed no cognitive improvement by methadone.

Given the current advances in assessing the brain network through rsFC generated from the times series of measured neurophysiological data (i.e., changes in cerebral blood flow, electrophysiological signals on scalp), the powerful neuroimaging tools in the review have high potentials to identify potential biomarkers and to generate both system-level and celllevel network topologies in OUD. Further, the traditional neuropsychological assessment tools have provided evidence of the change in certain brain functions and behaviors during abstinence in OUD. Considering that dysregulation of emotional processes and hyper-sensitivity to negative emotions in OUD patients even after prolonged abstinence, understanding the associations between maladaptive social interaction and the withdrawal syndromes in OUD are fundamentally important. After long-term cessation of opiates, withdrawal syndromes including generalized pain and discomfort and the psychological symptoms, such as restlessness and anxiety, start to merge and may stay from weeks to years (Schuckit, 2016). In addition to these distress during abstinence and social discrimination in the 
environment, it is not difficult to predict a relapse in an OUD abstinent patient whose reward MCL circuits and emotional mediating system have been disrupted resulting from chronic and intense opiate use-if an efficient diagnostic, a positive intervention and effective treatment programs fall apart.

In sum, when we address some limitations, we hope this review using multidisciplinary approach will provide a useful conceptual model of evaluating interventions and will shed light on the brain regional, functional and behavioral domains of OUD.

\section{AUTHOR CONTRIBUTIONS}

HI performed the literature review, wrote and contributed intellectually to the paper. $\mathrm{HI}$ and ZY read and approved the final manuscript.

\section{REFERENCES}

Bargh, J. A. (1989). "Conditional automaticity: varieties of automatic influence in social perception and cognition," in Unintended Thought, Vol. 3, eds J. S. Uleman and J. A. Bargh (New York, NY: The Guilford Press), 51-69.

Bart, G. (2012). Maintenance medication for opiate addiction: the foundation of recovery. J. Addict. Dis. 31, 207-225. doi: 10.1080/10550887.2012.694598

Bassett, D. S., Wymbs, N. F., Porter, M. A., Mucha, P. J., Carlson, J. M., and Grafton, S. T. (2011). Dynamic reconfiguration of human brain networks during learning. Proc. Natl. Acad. Sci. U.S.A. 108, 7641-7646. doi: $10.1073 /$ pnas. 1018985108

Baxter, M. G., Parker, A., Lindner, C. C. C., Izquierdo, A. D., and Murray, E. A. (2000). Control of response selection by reinforcer value requires interaction of amygdala and orbital prefrontal cortex. J. Neurosci. 20, 4311-4319. Available online at: http://www.jneurosci.org/content/20/11/4311.long

Chapman, D. P., Whitfield, C. L., Felitti, V. J., Dube, S. R., Edwards, V. J., and Anda, R. F. (2004). Adverse childhood experiences and the risk of depressive disorders in adulthood. J. Affect. Disord. 82, 217-225. doi: 10.1016/j.jad.2003.12.013

Crossley, N. A., Mechelli, A., Scott, J., Carletti, F., Fox, P. T., McGuire, P., et al. (2014). The hubs of the human connectome are generally implicated in the anatomy of brain disorders. Brain 137, 2382-2395. doi: 10.1093/brain/awu132

Crossley, N. A., Mechelli, A., Vértes, P. E., Winton-Brown, T. T., Patel, A. X., Ginestet, C. E., et al. (2013). Cognitive relevance of the community structure of the human brain functional coactivation network. Proc. Natl. Acad. Sci. U.S.A. 110, 11583-11588. doi: 10.1073/pnas.1220826110

Damoiseaux, J. S., and Greicius, M. D. (2009). Greater than the sum of its parts: a review of studies combining structural connectivity and resting-state functional connectivity. Brain Struct. Funct. 213, 525-533. doi: 10.1007/s00429-009-0208-6

Darke, S., Sims, J., McDonald, S., and Wickes, W. (2000). Cognitive impairment among methadone maintenance patients. Addiction 95, 687-695. doi: 10.1046/j.1360-0443.2000.9556874.x

Fair, D. A., Cohen, A. L., Dosenbach, N. U., Church, J. A., Miezin, F. M., Barch, D. M., et al. (2008). The maturing architecture of the brain's default network. Proc. Natl. Acad. Sci. U.S.A. 105, 4028-4032. doi: 10.1073/pnas.0800376105

Fair, D. A., Dosenbach, N. U., Church, J. A., Cohen, A. L., Brahmbhatt, S., Miezin, F. M., et al. (2007). Development of distinct control networks through segregation and integration. Proc. Natl. Acad. Sci. U.S.A. 104, 13507-13512. doi: 10.1073/pnas.0705843104

Fingelkurts, A. A., Fingelkurts, A. A., Kivisaari, R., Autti, T., Borisov, S., Puuskari, V., et al. (2006a). Increased local and decreased remote functional connectivity at EEG alpha and beta frequency bands in opioid-dependent patients. Psychopharmacology (Berl). 188, 42-52. doi: 10.1007/s00213-006-0474-4

Fingelkurts, A. A., Fingelkurts, A. A., Kivisaari, R., Autti, T., Borisov, S., Puuskari, V., et al. (2006b). Reorganization of the composition of brain oscillations and their temporal characteristics in opioid dependent

\section{FUNDING}

This article was supported by grants MYRG2014-00093FHS, MYRG 2015-00036-FHS, and MYRG2016-00110-FHS from the University of Macau in Macao SAR and grants FDCT 026/2014/A1 and FDCT 025/2015/A1 from the Macao government.

\section{ACKNOWLEDGMENTS}

We thank clinical psychologist Charles Zaroff for providing professional opinions before the article was constructed. We acknowledge the University of Macau in Macao SAR (MYRG2014-00093-FHS, MYRG 2015-00036-FHS and MYRG2016-00110-FHS) and the Macao government (FDCT 026/2014/A1 and FDCT 025/2015/A1) for financial support.

patients. Prog. Neuropsychopharmacol. Biol. Psychiatry 30, 1453-1465. doi: 10.1016/j.pnpbp.2006.06.005

Fingelkurts, A. A., Fingelkurts, A. A., Kivisaari, R., Autti, T., Borisov, S., Puuskari, V., et al. (2007). Opioid withdrawal results in an increased local and remote functional connectivity at EEG alpha and beta frequency bands. Neurosci. Res. 58, 40-49. doi: 10.1016/j.neures.2007.01.011

Fingelkurts, A. A., Fingelkurts, A. A., Kivisaari, R., Autti, T., Borisov, S., Puuskari, V., et al. (2009). Methadone restores local and remote EEG functional connectivity in opioid-dependent patients. Int. J. Neurosci. 119, 1469-1493. doi: 10.1080/00207450903007985

Fornito, A., Zalesky, A., and Bullmore, E. (2016). Fundamentals of Brain Network Analysis. San Diego, CA: Academic Press.

Franken, I. H., Stam, C. J., Hendriks, V. M., and van den Brink, W. (2004). Electroencephalographic power and coherence analyses suggest altered brain function in abstinent male heroin-dependent patients. Neuropsychobiology 49, 105-110. doi: 10.1159/000076419

Fries, P., Neuenschwander, S., Engel, A. K., Goebel, R., and Singer, W. (2001). Rapid feature selective neuronal synchronization through correlated latency shifting. Nat. Neurosci. 4, 194-200. doi: 10.1038/84032

Gerra, G., Somaini, L., Manfredini, M., Raggi, M., Saracino, M., Amore, M., et al. (2014). Dysregulated responses to emotions among abstinent heroin users: correlation with childhood neglect and addiction severity. Prog. Neuropsychopharmacol. Biol. Psychiatry 48, 220-228. doi: 10.1016/j.pnpbp.2013.10.011

Gerstein, G. L., and Perkel, D. H. (1969). Simultaneously recorded trains of action potentials: analysis and functional interpretation. Science 164, 828-830.

Gorricho, M. D., and Usón, J. R. V. (2008). QEEG mapping and methadone. Eur. J. Psychiatry 22, 29-37. Available online at: http://scielo.isciii.es/scielo. php?script $=$ sci_arttext\&pid $=$ S0213-61632008000100004\&lng $=$ en $\& n r m=$ isoISSN0213-6163

Goldstein, R. Z., and Volkow, N. D. (2002). Drug addiction and its underlying neurobiological basis: neuroimaging evidence for the involvement of the frontal cortex. Am. J. Psychiatry 159, 1642-1652. doi: 10.1176/appi.ajp.159.10.1642

Goldstein, R. Z., and Volkow, N. D. (2011). Dysfunction of the prefrontal cortex in addiction: neuroimaging findings and clinical implications. Nat. Rev. Neurosci. 12, 652-669. doi: 10.1038/nrn3119

Greicius, M. D., Supekar, K., Menon, V., and Dougherty, R. F. (2009). Restingstate functional connectivity reflects structural connectivity in the default mode network. Cereb. Cortex 19, 72-78. doi: 10.1093/cercor/bhn059

Hebb, D. O. (2005). The Organization of Behavior: A Neuropsychological Theory. Mahwah, NJ: Psychology Press.

Honey, C., Sporns, O., Cammoun, L., Gigandet, X., Thiran, J.-P., Meuli, R., et al. (2009). Predicting human resting-state functional connectivity from structural connectivity. Proc. Natl. Acad. Sci. U.S.A. 106, 2035-2040. doi: 10.1073/pnas.0811168106 
Hou, Y., Zhao, L., Yao, Q., and Ding, L. (2016). Altered economic decision-making in abstinent heroin addicts: evidence from the ultimatum game. Neurosci. Lett. 627, 148-154. doi: 10.1016/j.neulet.2016.06.002

Ieong, H. F.-h. (2013). "Literature review craving: the neurotoxic and socialcognitive effects of drugs consumptions of adults, adolescences, including prenatal exposure to drugs of abuse," in Proceedings of the 8th Mainland, Hong Kong and Macau Conference on Prevention of Drug Abuse (Macau).

Ieong, H. F.-h. (2015). Application of Functional Near-Infrared Spectroscopy to Study Resting-State Functional Brain Connectivity in Addiction. Paper presented at the Bioimaging Asia-Biological Imaging, Singapore.

Knyazeva, M. G., and Innocenti, G. M. (2001). EEG coherence studies in the normal brain and after early-onset cortical pathologies. Brain Res. Rev. 36, 119-128. doi: 10.1016/S0165-0173(01)00087-X

Levi, J., Segal, L., and Kohn, D. (2012). The Facts Hurt: A State-by-State Injury Prevention Policy Report. Washington, DC: Trust for America's Health.

Li, Y., Liu, Y., Li, J., Qin, W., Li, K., Yu, C., et al. (2009). Brain anatomical network and intelligence. PLoS Comput. Biol. 5:e1000395. doi: 10.1371/journal.pcbi.1000395

Liu, J., Liang, J., Qin, W., Tian, J., Yuan, K., Bai, L., et al. (2009). Dysfunctional connectivity patterns in chronic heroin users: an fMRI study. Neurosci. Lett. 460, 72-77. doi: 10.1016/j.neulet.2009.05.038

Ma, N., Liu, Y., Fu, X.-M., Li, N., Wang, C.-X., Zhang, H., et al. (2011). Abnormal brain default-mode network functional connectivity in drug addicts. PLOS ONE 6:e16560. doi: 10.1371/journal.pone.0016560

Ma, N., Liu, Y., Li, N., Wang, C.-X., Zhang, H., Jiang, X.-F., et al. (2010). Addiction related alteration in resting-state brain connectivity. Neuroimage 49, 738-744. doi: 10.1016/j.neuroimage.2009.08.037

Miller, L. (1985). Neuropsychological assessment substance abusers: review and recommendations. J. Subst. Abuse Treat. 2, 5-17. doi: 10.1016/0740-5472(85)90017-0

Mintzer, M. Z., Copersino, M. L., and Stitzer, M. L. (2005). Opioid abuse and cognitive performance. Drug Alcohol Depend. 78, 225-230. doi: 10.1016/j.drugalcdep.2004.10.008

Mintzer, M. Z., and Stitzer, M. L. (2002). Cognitive impairment in methadone maintenance patients. Drug Alcohol Depend. 67, 41-51. doi: 10.1016/S0376-8716(02)00013-3

Morie, K. P., Garavan, H., Bell, R. P., De Sanctis, P., Krakowski, M. I., and Foxe, J. J. (2014). Intact inhibitory control processes in abstinent drug abusers (II): a high-density electrical mapping study in former cocaine and heroin addicts. Neuropharmacology 82, 151-160. doi: 10.1016/j.neuropharm.2013.02.023

Nestler, E. J. (2005). Is there a common molecular pathway for addiction? Nat. Neurosci. 8, 1445-1449. doi: 10.1038/nn1578

Noel, M. B., and Gratton, A. (1995). Electrochemical evidence of increased dopamine transmission in prefkontal cortex and nucleus accumbens elicited by ventral tegmental $\mu$-opioid receptor activation in freely behaving rats. Synapse 21, 110-122. doi: 10.1002/syn.890210204

Parvaz, M. A., Alia-Klein, N., Woicik, P. A., Volkow, N. D., and Goldstein, R. Z. (2011). Neuroimaging for drug addiction and related behaviors. Rev. Neurosci. 22, 609-624. doi: 10.1515/RNS.2011.055

Passetti, F., Clark, L., Mehta, M., Joyce, E., and King, M. (2008). Neuropsychological predictors of clinical outcome in opiate addiction. Drug Alcohol Depend. 94, 82-91. doi: 10.1016/j.drugalcdep.2007.10.008

Pezawas, L., Meyer-Lindenberg, A., Drabant, E. M., Verchinski, B. A., Munoz, K. E., Kolachana, B. S., et al. (2005). 5-HTTLPR polymorphism impacts human cingulate-amygdala interactions: a genetic susceptibility mechanism for depression. Nat. Neurosci. 8, 828-834. doi: 10.1038/nn1463

Phillips, M. L., Drevets, W. C., Rauch, S. L., and Lane, R. (2003). Neurobiology of emotion perception I: the neural basis of normal emotion perception. Biol. Psychiatry 54, 504-514. doi: 10.1016/S0006-3223(03)00168-9

Reilly, P. M., Sees, K. L., Shopshire, M. S., Hall, S. M., Delucchi, K. L., Tusel, D. J., et al. (1995). Self-efficacy and illicit opioid use in a 180day methadone detoxification treatment. J. Consult. Clin. Psychol. 63:158. doi: 10.1037/0022-006X.63.1.158

Schuckit, M. A. (2016). Treatment of opioid-use disorders. N. Engl. J. Med. 375, 357-368. doi: 10.1056/NEJMra1604339
Singer, W. (1999). Neuronal synchrony: a versatile code for the definition of relations? Neuron 24, 49-65. doi: 10.1016/S0896-6273(00) 80821-1

Singer, W. (2013). Cortical dynamics revisited. Trends Cogn. Sci. 17, 616-626. doi: 10.1016/j.tics.2013.09.006

Sporns, O., Chialvo, D. R., Kaiser, M., and Hilgetag, C. C. (2004). Organization, development and function of complex brain networks. Trends Cogn. Sci. 8, 418-425. doi: 10.1016/j.tics.2004.07.008

Stein, J. L., Wiedholz, L. M., Bassett, D. S., Weinberger, D. R., Zink, C. F., Mattay, V. S., et al. (2007). A validated network of effective amygdala connectivity. Neuroimage 36, 736-745. doi: 10.1016/j.neuroimage.2007.03.022

Sutherland, M. T., McHugh, M. J., Pariyadath, V., and Stein, E. A. (2012). Resting state functional connectivity in addiction: lessons learned and a road ahead. Neuroimage 62, 2281-2295. doi: 10.1016/j.neuroimage.2012.01.117

United Nations Office on Drugs and Crime (2014). World Drug Report 2014. New York, NY: United Nations. Available online at: http://www.unodc.org/wdr2014/

Wang, P.-W., Lin, H.-C., Liu, G.-C., Yang, Y.-H. C., Ko, C.-H., and Yen, C.-F. (2016). Abnormal interhemispheric resting state functional connectivity of the insula in heroin users under methadone maintenance treatment. Psychiatry Res. 255, 9-14. doi: 10.1016/j.pscychresns.2016.07.009

Womelsdorf, T., Valiante, T. A., Sahin, N. T., Miller, K. J., and Tiesinga, P. (2014). Dynamic circuit motifs underlying rhythmic gain control, gating and integration. Nat. Neurosci. 17, 1031-1039. doi: 10.1038/nn.3764

Xie, C., Li, S.-J., Shao, Y., Fu, L., Goveas, J., Ye, E., et al. (2011). Identification of hyperactive intrinsic amygdala network connectivity associated with impulsivity in abstinent heroin addicts. Behav. Brain Res. 216, 639-646. doi: 10.1016/j.bbr.2010.09.004

Yan, W.-S., Li, Y.-H., Xiao, L., Zhu, N., Bechara, A., and Sui, N. (2014). Working memory and affective decision-making in addiction: a neurocognitive comparison between heroin addicts, pathological gamblers and healthy controls. Drug Alcohol Depend. 134, 194-200. doi: 10.1016/j.drugalcdep.2013.09.027

Yuan, K., Qin, W., Dong, M., Liu, J., Liu, P., Zhang, Y., et al. (2010). Combining spatial and temporal information to explore resting-state networks changes in abstinent heroin-dependent individuals. Neurosci. Lett. 475, 20-24. doi: 10.1016/j.neulet.2010.03.033

Zalesky, A., Fornito, A., Seal, M. L., Cocchi, L., Westin, C.-F., Bullmore, E. T., et al. (2011). Disrupted axonal fiber connectivity in schizophrenia. Biol. Psychiatry 69, 80-89. doi: 10.1016/j.biopsych.2010.08.022

Zhai, T., Shao, Y., Chen, G., Ye, E., Ma, L., Wang, L., et al. (2015). Nature of functional links in valuation networks differentiates impulsive behaviors between abstinent heroin-dependent subjects and nondrug-using subjects. Neuroimage 115, 76-84. doi: 10.1016/j.neuroimage.2015.04.060

Zhang, Y., Tian, J., Yuan, K., Liu, P., Zhuo, L., Qin, W., et al. (2011). Distinct resting-state brain activities in heroin-dependent individuals. Brain Res. 1402, 46-53. doi: 10.1016/j.brainres.2011.05.054

Zhao, L.-Y., Zhang, X.-L., Shi, J., Epstein, D. H., and Lu, L. (2009). Psychosocial stress after reactivation of drug-related memory impairs later recall in abstinent heroin addicts. Psychopharmacology (Berl). 203, 599-608. doi: 10.1007/s00213-008-1406-2

Zhou, Y., Zhu, H., Jin, X., Li, X., Zhang, M., Zhang, F., et al. (2012). Biased attention towards negative schematic expression in abstinent heroin abusers. J. Behav. Ther. Exp. Psychiatry 43, 705-710. doi: 10.1016/j.jbtep.2011. 10.004

Conflict of Interest Statement: The authors declare that the research was conducted in the absence of any commercial or financial relationships that could be construed as a potential conflict of interest.

Copyright (c) 2017 Ieong and Yuan. This is an open-access article distributed under the terms of the Creative Commons Attribution License (CC BY). The use, distribution or reproduction in other forums is permitted, provided the original author(s) or licensor are credited and that the original publication in this journal is cited, in accordance with accepted academic practice. No use, distribution or reproduction is permitted which does not comply with these terms. 